\title{
Blood-brain barrier permeability is positively correlated with cerebral microvascular perfusion in the early fluid percussion-injured brain of the rat
}

\author{
Yong Lin ${ }^{1, *}$, Yaohua Pan ${ }^{1, *}$, Mingliang Wang ${ }^{2}$, Xianjian Huang ${ }^{1}$, Yuhua Yin ${ }^{1}$, Yu Wang ${ }^{1}$, Feng Jia ${ }^{1}$, \\ Wenhao Xiong ${ }^{1}$, Nu Zhang ${ }^{3}$ and Ji-yao Jiang ${ }^{1}$
}

The blood-brain barrier (BBB) opening following traumatic brain injury (TBI) provides a chance for therapeutic agents to cross the barrier, yet the reduction of the cerebral microvascular perfusion after TBI may limit the intervention. Meanwhile, optimizing the cerebral capillary perfusion by the strategies such as fluid administration may cause brain edema due to the BBB opening post trauma. To guide the TBI therapy, we characterized the relationship between the changes in the cerebral capillary perfusion and BBB permeability after TBI. First, we observed the changes of the cerebral capillary perfusion by the intracardiac perfusion of Evans Blue and the BBB disruption with magnetic resonance imaging (MRI) in the rat subjected to lateral fluid percussion (FP) brain injury. The correlation between two variables was next evaluated with the correlation analysis. Since related to BBB breakdown, matrix metalloproteinase-9 (MMP-9) activity was finally detected by gelatin zymography. We found that the ratios of the perfused microvessel numbers in the lesioned cortices were significantly reduced at 0 and $1 \mathrm{~h}$ post trauma compared with that in the normal cortex, which then dramatically recovered at 4 and $24 \mathrm{~h}$ after injury, and that the BBB permeability was greatly augmented in the ipsilateral parts at 4,12 , and $24 \mathrm{~h}$, and in the contralateral area at $24 \mathrm{~h}$ after injury compared with that in the uninjured brain. The correlation analysis showed that the BBB permeability increase was related to the restoration of the cerebral capillary perfusion over a 24-h period post trauma. Moreover, the gelatin zymography analysis indicated that the MMP-9 activity in the injured brain increased at $4 \mathrm{~h}$ and significantly elevated at 12 and $24 \mathrm{~h}$ as compared to that at 0 or $1 \mathrm{~h}$ after TBI. Our findings demonstrate that the $4 \mathrm{~h}$ post trauma is a critical turning point during the development of TBI, and, importantly, the correlation analysis may guide us how to treat TBI.

Laboratory Investigation (2012) 92, 1623-1634; doi:10.1038/labinvest.2012.118; published online 10 September 2012

KEYWORDS: blood-brain barrier; MRI; rat; traumatic brain injury; vascular reactivity

After traumatic brain injury (TBI), brain tissues undergo primary injury and consequent secondary injury. ${ }^{1,2}$ The primary injury is caused by mechanical forces at the moment of the impact, resulting in various tissue damages, such as brain structure disorders and blood vessel injury. ${ }^{3}$ The secondary injury, occurring minutes or hours to days post trauma, is a result of the combination of biochemical and pathophysiological responses, including hypo- or hyperperfusion, edema, and inflammation. ${ }^{4-6}$ The secondary injury could cause the neuron loss and subsequent dysfunction of the central nervous system (CNS). Therefore, the sooner clinical interventions are introduced, the better chance that the secondary injury can be prevented. However, the therapeutic efficacy is often constrained by blood-brain barrier (BBB).

The $\mathrm{BBB}$ is a physical barrier that separates circulating blood and brain extracellular fluid. It consists of endothelial cells, basal lamina, and astrocytic foot processes, and is also sealed by tight junctions. ${ }^{7,8}$ The studies using the intravenous injection of the tracers, including Evans Blue ${ }^{9,10}$ and horseradish peroxidase, ${ }^{11,12}$ in the animal models of TBI have demonstrated that the $\mathrm{BBB}$ is disrupted within hours or

\footnotetext{
${ }^{1}$ Department of Neurological Surgery, Renji Hospital, School of Medicine, Shanghai Jiao Tong University, Shanghai, People's Republic of China; ${ }^{2}$ Department of Radiology, Ruijin Hospital, School of Medicine, Shanghai Jiao Tong University, Shanghai, People's Republic of China and ${ }^{3}$ Department of Neurological Surgery, Second People's Hospital, Wenzhou Medical University, Wenzhou, Zhejiang, People's Republic of China

Correspondence: Professor J-Y Jiang, MD, PhD, Department of Neurological Surgery, Renji Hospital, School of Medicine, Shanghai Jiao Tong University, 1630 Dongfang Road, Shanghai 200127, People's Republic of China.

"These authors contributed equally to this work.

Received 14 May 2012; revised 27 June 2012; accepted 28 June 2012
} 
days after injury. Although these evaluations provide the histological evidence about the disruption of the BBB post trauma, the barrier permeability examination by using the clinically relevant techniques, like magnetic resonance imaging (MRI), is more meaningful to patients. It has been reported that the alterations of the $\mathrm{BBB}$ permeability evaluated by MRI vary in the different animal models of TBI. ${ }^{13,14}$ In the rat model of lateral fluid percussion (FP) brain injury, the MRI observation of the $\mathrm{BBB}$ permeability reveals the barrier breakdown within $12 \mathrm{~h}$ post trauma, ${ }^{14}$ but few studies have been performed to investigate the longer events, such as the changes over a 24 -h period post trauma, of the barrier permeability with MRI.

The disruption of the BBB after injury, which may be related to matrix metalloproteinase-9 (MMP-9) activity, ${ }^{10,15}$ offers the possibility for the molecules with heavy molecular weight, like nerve growth factor, to cross the barrier to treat TBI patients. However, the compromised cerebral blood flow (CBF) following $\mathrm{TBI}^{16,17}$ may hamper the intravenously administered molecules to reach the lesioned areas of the brain and further reduce the efficacy of the treatment.

In the clinic and laboratory, the changes of the CBF can be detected by using different techniques, such as Doppler, single photon emission computed tomography (SPECT), and MRI. ${ }^{16-18}$ In contrast, the intravenous administration of the tracers like the Evans Blue can histologically assess cerebral microvascular perfusion, ${ }^{19,20}$ but the disadvantage of this intravenous injection is that the different circulation periods of the tracer may result in the different numbers of the perfused capillary in impacted areas. ${ }^{21}$

Several therapeutic interventions, such as fluid and medicine administration, have been reported to ameliorate the CBF and optimize the cerebral capillary perfusion, ${ }^{6,22}$ which are able to prevent the brain ischemia, reduce the secondary brain injury, and improve the neurological outcome after TBI. The therapy of the fluid administration involves maintaining effective blood volume in the circulation system to ensure adequate cerebral perfusion pressure (CPP) and delivering medical molecules to the injured sites. As the BBB is impaired post trauma, improved microvascular perfusion following the fluid administration may cause water to filter out into the surrounding tissue and further exacerbate the edema in brain parenchyma. ${ }^{6,23}$

There are few data documenting how the capillary perfusion correlates with the BBB permeability following injury. However, the resolution may guide us not only how to optimize the cerebral capillary perfusion but also not to cause other neurological pathophysiologies such as the edema and increased intracranial pressure (ICP). In this study, our objective is to address the relationship between the changes in the cerebral capillary perfusion and the BBB permeability after TBI. We investigated the alterations of the cerebral capillary perfusion by intracardially perfusing Evans Blue and a 24-h period event of the BBB disruption by MRI in the lateral FP brain injury model of TBI in the rat. The corre- lation between two variables was evaluated with correlation analysis. Moreover, the MMP-9 activities during a period of $24 \mathrm{~h}$ after injury were assessed by gelatin zymography.

\section{MATERIALS AND METHODS Animals}

This study was approved by Shanghai Jiao Tong University School of Medicine and performed in accordance with the Guide for Laboratory Animal Care and Use. Male SpragueDawley rats, weighing 200-320 g and purchased from Shanghai SLAC and Sino-British Animal facilities (Shanghai, China), were selected randomly for this experiment. The rats were fed in the cages, with food and drinkable water provided ad libitum, at room temperature in a $12 \mathrm{~h}$ light/dark cycle.

\section{Lateral FP Brain Injury}

Lateral FP brain injury was performed as described previously by McIntosh et al. ${ }^{24}$ In brief, each animal was intraperitoneally anesthetized with $0.4 \%$ sodium pentobarbital at a dose of $40 \mathrm{mg} / \mathrm{kg}$. The rat was placed in a stereotaxic frame with a head holder. A $37^{\circ} \mathrm{C}$, heating pad was used throughout the surgery. A midline incision between the eyes and the ears was made on the sterilized scalp. After the tissues on the skull were cleaned, a circle craniotomy, over the left parietal cortex between the bregma and the lambda suture, was performed with a $4.7-\mathrm{mm}$-diameter Michele Trephine 3/16 (Miltex, No. 26-140, Germany). The bone was removed, but the dura mater was kept intact. Thereafter, two screws were placed at the rostral and caudal sites of the craniotomy. A hub connector was inserted into the craniotomy and fixed with the screws using a dental cement. At 30-60 min after the hub placement, the hub was filled with sterile isotonic saline and then connected to the FP injury device (Virginia Commonwealth University Medical Center, Richmond, VA, USA). The FP brain injury was generated by releasing the pendulum hammer at an angle of $12.5^{\circ}(<1 \mathrm{~atm})$ to strike the plunger of the cylinder filled with sterilized water.

\section{Evaluation of the Cerebral Microvascular Perfusion}

The cerebral capillary perfusion before and after lateral FP injury was determined by a modified technique of the intracardiac perfusion of the paraformaldehyde (PFA) fixative solution supplemented with Evans Blue, which was used by del Valle et $^{2} \mathrm{l}^{25}$ to measure the BBB breakdown after cryoinjury in mice, combined with the total blood vessel staining by an alkaline phosphatase (AP) method ${ }^{19,26}$ in the rat.

The animals were divided into naïve group and the groups of $0,1,4,12$, and $24 \mathrm{~h}$ post trauma. Four in each group. At each time point, the rats were intracardially perfused with prewarmed $0.9 \% \mathrm{NaCl}$ briefly to wash out blood cells, followed by $0.5 \%$ Evans Blue in cold $4 \%$ PFA. The perfusion solution was positioned at a height of approximately $100 \mathrm{~cm}$ above the perfused animal, which generated a mean arterial pressure (MAP)-like pressure of $73.56 \mathrm{~mm} \mathrm{Hg}\left(100 \mathrm{~cm} \cdot \mathrm{H}_{2} \mathrm{O}\right)$. The normal ICP of the rat is about $4.12 \mathrm{~mm} \mathrm{Hg}, 27,28$ so the CPP 
produced in our study $(\mathrm{CPP}=\mathrm{MAP}-\mathrm{ICP})$ was at the value of approximately $69.44 \mathrm{~mm} \mathrm{Hg}$, close to the normal CPP value of $70 \mathrm{~mm} \mathrm{Hg}$ in the rat. ${ }^{29}$

After perfusion, the brain was taken out, post-fixed in $4 \%$ PFA at $4{ }^{\circ} \mathrm{C}$ for $4 \mathrm{~h}$, and then cryoprotected in 20 and $30 \%$ sucrose solutions in PBS at $4{ }^{\circ} \mathrm{C}$ for 3 days. The brain tissues were cut $40 \mu \mathrm{m}$ in thickness in a cryostat (Leica Microsystems, Wetzlar, Germany). Sections were mounted on gel-coated slides, dried at $37^{\circ} \mathrm{C}$ for $1 \mathrm{~h}$, and kept at $-20^{\circ} \mathrm{C}$ for use.

The Evans Blue-perfused cerebral microvessels in the sections, which exhibit fluorescence when the Evans Blue binds to proteins, were examined and imaged at each time point in the ischemic centers of the lesioned cortices of the rat brains using a Leica fluorescence microscope equipped with a CCD camera (Leica Microsystems). Afterwards, the same sections were stained with AP method for the blood vessels as follows: the slides were rinsed in distilled water, incubated with a fresh and prewarmed reaction solution (3.8 g/l Fast Blue RR, $0.5 \mathrm{~g} / 1 \alpha$-naphthyl phosphate, $3.8 \mathrm{~g} / \mathrm{l}$ sodium metaborate, and $1.7 \mathrm{~g} / \mathrm{l}$ magnesium sulfate in distilled water) at $37^{\circ} \mathrm{C}$ for $15 \mathrm{~min}$, and then washed in distilled water. The fields used for evaluating the Evans Blue-perfused blood vessels were relocated and photographed again. Both the Evans Blue-perfused and the AP-stained microvessels were quantified on the optical and fluorescence images. Only a blood vessel with clear morphology and with a diameter $<12 \mu \mathrm{m}$ was counted. The spot in the section was excepted as a transverse capillary to avoid false count or double counts for an identical capillary loop. The ratio between the number of Evans Blue-perfused capillary and AP-stained capillary amount was used for evaluating the cerebral capillary perfusion.

\section{Measurement of the BBB Permeability \\ Histological analysis of the mechanical injury-caused primary $B B B$ breakdown}

The disruption of the BBB caused by the primary mechanical injury was also assessed at $0 \mathrm{~h}$ post trauma in the Evans Blueperfused rat. The primary $\mathrm{BBB}$ breakdown, reflected by the leakage of the Evans Blue, was evaluated with the Leica fluorescence microscope equipped with a CCD camera (Leica Microsystems).

\section{MRI Methodology for the time course of the BBB breakdown in the early injured brain}

To observe the changes of the BBB breakdown over a 24-h period after TBI, MRI was performed on a 3.0T MRI system (Signa HDx; GE Medical System, Milwaukee, WI, USA). Because contrast agent Gd-DTPA (Magnevist, Schering (Guangzhou), Guangzhou, China) is unable to cross the normal BBB, the signal intensity enhancement on the MRI images post contrast reflects the BBB disruption.

Each of six rats was anesthetized before injury as well as at $1,4,12$, and $24 \mathrm{~h}$ post trauma, and then placed in a prone position on a custom-made $5-\mathrm{cm}$ diameter transmit-receiving birdcage coil, parallel to the B0 magnetic field, in the magnet bore. At each time point, the rat was conducted transverse high spatial-resolution $\mathrm{T}_{2^{-}}$and $\mathrm{T}_{1}$-weighted MRI scans over the head before and $\mathrm{T}_{1}$-weighted MRI scans right after the injection of $0.6 \mathrm{ml}$ of $1: 1 \quad(\mathrm{v} / \mathrm{v})$ diluted $469.01 \mathrm{mg} / \mathrm{ml}$ Gd-DTPA (Magnevist) with $0.9 \% \mathrm{NaCl}$ via tail vein.

The MRI images were acquired with the following parameters: for $T_{2} W I$, repetition rate $(T R)=2900 \mathrm{~ms}$, echo time $(\mathrm{TE})=80 \mathrm{~ms}$, matrix: $224 \times 192, \mathrm{NEX}=6$, echo train length $(\mathrm{ETL})=16$, bandwidth $=31.25 \mathrm{kHz}$, field of view $(\mathrm{FOV})=$ $6.0 \times 4.8 \mathrm{~cm}^{2}$, slice thickness $=1.2 \mathrm{~mm}$, intersection gap $=0.2$ $\mathrm{mm}$; for $\mathrm{T}_{1} \mathrm{WI}, \mathrm{TR}=600 \mathrm{~ms}, \mathrm{TE}=12 \mathrm{~ms}$, matrix: $256 \times 192$, $\mathrm{NEX}=3, \mathrm{ETL}=4$, bandwidth $=31.25 \mathrm{kHz}, \quad \mathrm{FOV}=6.0 \times 4.8$ $\mathrm{cm}^{2}$, slice thickness $=1.2 \mathrm{~mm}$, intersection gap $=0.2 \mathrm{~mm}$.

The signal intensities on the MRI images from the lesioned brains were quantified using densitometric analyses with the NIH ImageJ software (ImageJ 1.43u; National Institutes of Health, USA). The optical density of the cortex and hippocampus part in the ipsilateral hemisphere of the rat brain was measured, so was that in the contralateral hemisphere. Relative optical density was determined by the cortex and hippocampus density divided by the background density of the image. The alteration of the signal intensity was defined by the difference between pre- and post-contrast relative optical densities.

\section{Gelatin Zymography}

In the uninjured group and the groups of $0,1,4,12$, and $24 \mathrm{~h}$ post trauma, the rat brains of four in each group were quickly removed and stored at $-20^{\circ} \mathrm{C}$ for MMP-9 analysis. After dissected to $12 \mathrm{~mm}$ in thickness, centered over the impacted site, each brain tissue was further separated to the ipsilateral and contralateral parts. The tissue was homogenized in a cold lysis buffer (50 mM Tris- $\mathrm{HCl}, \mathrm{pH}$ 8.0, $150 \mathrm{mM} \mathrm{NaCl}, 1 \%$ NP-40, $0.5 \%$ deoxycholate, and $0.1 \%$ SDS) at a ratio of 1:6 (w/v) and centrifuged at $25150 \mathrm{~g}$ for $15 \mathrm{~min}$. The supernatant was saved and further diluted with the cold lysis buffer at a ratio of $1: 4(\mathrm{v} / \mathrm{v})$ for protein concentration measurement by using BCA protein assay kit (Pierce, Rockford, IL, USA). Equal amounts and volumes $(20 \mu \mathrm{g} / 20 \mu \mathrm{l})$ of the total proteins were loaded and separated on a $10 \%$ Tris-glycine gel with $0.1 \%$ gelatin. After electrophoresis, the gels were washed in $2.7 \%(\mathrm{w} / \mathrm{v})$ Triton X-100 for $30 \mathrm{~min}$ and equilibrated in a developing buffer (50 mM Tris- $\mathrm{HCl}, \mathrm{pH} 7.5,10 \mathrm{mM} \mathrm{CaCl}_{2}$, and $0.01 \%$ sodium azide) for another $30 \mathrm{~min}$. The MMP-9 activities were visualized by Coomassie blue staining after the gels were incubated in the developing buffer for 4-5 days at $37^{\circ} \mathrm{C}$.

The MMP-9 activities were quantified by densitometric analyses with the NIH ImageJ software (ImageJ 1.43u, National Institutes of Health). The optical densities were measured for each band and its background within the same lane. The difference between the densities of each band and its background was determined as the density of the MMP-9 activity. Relative optical density was defined by the MMP-9 
activity density in the lesioned brain divided by the one in the normal brain.

\section{Statistical Analysis}

The data of the ratio of the Evans Blue-perfused capillary number to AP-stained capillary amount and the MMP-9 activity intensity were analyzed with one-way analysis of variance (ANOVA), followed by Tukey and Games-Howell post-hoc tests, respectively, in the Statistical Package for Social Sciences 13.0 (SPSS, Chicago, IL, USA). The MRI signal intensity data in the different groups were compared using one-way ANOVA with repeated measures, followed by the least significant difference (LSD) post-hoc test because a Mauchly's test of sphericity showed no statistically significant differences $(P=0.53)$ in the SPSS. The data were also analyzed by Student's $t$-test with two tails.

Although the rats used for the analyses of the perfused capillary and the BBB disruption were different, they were from the same strain and randomly selected for the experiments. Therefore, we pooled the data for correlation analysis. The correlation between the changes of the ratio of the perfused capillary number and the MRI signal intensity post-trauma was determined with the Pearson correlation test in the SPSS.

Statistical significance was defined at $P \leq 0.05$. The data were presented as the means \pm standard errors of the means (s.e.m.).

\section{RESULTS}

\section{Evans Blue Labels Cerebral Blood Vessels with Dyes}

In either the uninjured or the injured rat, the cerebral blood vessel wall was stained with the Evans Blue, which was seen as a clear light blue layer as observed with an optical microscope (Figure 1a and b). Differently, there was another uneven layer in dark blue surrounding the light blue wall of the blood vessel in the injured brain, which was considered as a consequence of bleeding due to the existence of the blood cells outside the vessel, similar to those inside the vessel (Figure 1d). Under a fluorescence microscope, while the vessel walls in both the normal and the injured brains exhibited weaker red fluorescence (Figure $1 \mathrm{~b}$ and $\mathrm{e}$ ), the fluorescence is stronger in the bleeding layer outside the blood vessel of the injured brain (Figure 1e). It suggests that the Evans Blue can exhibit strong red fluorescence when binding to the leaked blood, which was confirmed by Figure $1 \mathrm{~g}$ and $\mathrm{h}$. There was slight fluorescence around the vessel in the normal brain (Figure 1b), which might be a result of the Evans Blue dye diffusion out of the blood vessel due to the air drying of the sections. ${ }^{19}$ The images a and b, d and $\mathrm{e}$, and $\mathrm{g}$ and $\mathrm{h}$ are digitally combined in the images $\mathrm{c}, \mathrm{f}$, and $i$, respectively, to show colocalization.

\section{Cerebral Microvascular Responses to TBI \\ Compromised cerebral capillary perfusion}

In the uninjured brain, the Evans Blue-perfused cerebral capillaries showed clear vascular morphology under the fluorescence microscope (Figure 2a). In contrast, at $0,1,4$, 12 , and $24 \mathrm{~h}$ after FP injury, the tracer-labeled blood vessels in the injured cortices disappeared or reduced in quantities (Figure $2 \mathrm{~b}-\mathrm{f}$ ), although the AP staining showed that the blood vessels still existed at the injured sites (Figure 2g-i for $4 \mathrm{~h}$, data at other time points not shown).

The ratio of the Evans Blue-perfused capillary number to the number of AP-stained blood vessels in the lesioned cortex right after (at $0 \mathrm{~h}$ after) FP injury was significantly reduced compared with that in the normal cortex (Figure 3, $P=0.005$ by Tukey's post-hoc test). The great reduction continued to 1 h post trauma (Figure 3, $P=0.023$ by Tukey's post-hoc test). However, at $4 \mathrm{~h}$ after FP injury, the decreased fraction post trauma was significantly increased in comparison with that at $0 \mathrm{~h}$ after injury (Figure 3, $P=0.016$ by Student's $t$-test). This dramatic improvement in the ratio of the perfused capillary number was kept to $24 \mathrm{~h}$ post trauma as compared to that at $0 \mathrm{~h}$ after TBI (Figure 3, $P=0.017$ by Student's $t$-test).

\section{Impaired BBB}

The permeability of the BBB was impaired after FP injury.

Primary mechanical injury induces the primary $B B B$ breakdown The primary breakdown of the BBB was detected immediately after the primary mechanical injury. In the uninjured brain, the Evans Blue clearly stained the cerebral blood vessels as observed under both the optical (Figure 4a) and the fluorescence (Figure $4 \mathrm{~b}$ ) microscopes. In contrast, at the nonhemorrhagic sites of the injured cortex right after TBI (Figure 4c), stronger Evans Blue fluorescence was noted (Figure 4d). The extravasation of the tracer indicates that the $\mathrm{BBB}$ permeability is increased right after TBI.

\section{$M R I$ monitors the BBB breakdown in the early FP lesioned brain}

The $\mathrm{T}_{2}$-weighted MRI images clearly exhibited the edematous and lesioned regions in the ipsilateral cortices of the injured brains at $1,4,12$, and $24 \mathrm{~h}$ after TBI (Figure $5 \mathrm{a}$ ). There were the background signals in both the uninjured and the injured brains on the pre-contrast $\mathrm{T}_{1}$-weighted MRI images, except that slight enhancement was observed at the injured site of the brain at $4 \mathrm{~h}$ post trauma. On the contrary, the Gd-DTPA leakages in the brains were obviously noted after TBI on the post-contrast $\mathrm{T}_{1}$-weighted scans (Figure $5 \mathrm{a}$ ). The signal intensities were significantly elevated in the ipsilateral cortices and hippocampi of the contused brains at 4,12 , and $24 \mathrm{~h}$ post trauma compared with that in the uninjured brain (Figure $5 \mathrm{a}$ and $\mathrm{b}, P=0.021,0.013$, and 0.027 by repeated measures ANOVA, respectively). Moreover, an enhancement of the signal intensity was significantly greater in the contralateral cortex and hippocampus at $24 \mathrm{~h}$ after injury in comparison to that in the uninjured brain (Figure $5 \mathrm{a}$ and $\mathrm{c}, P=0.05$ by Student's $t$-test). 

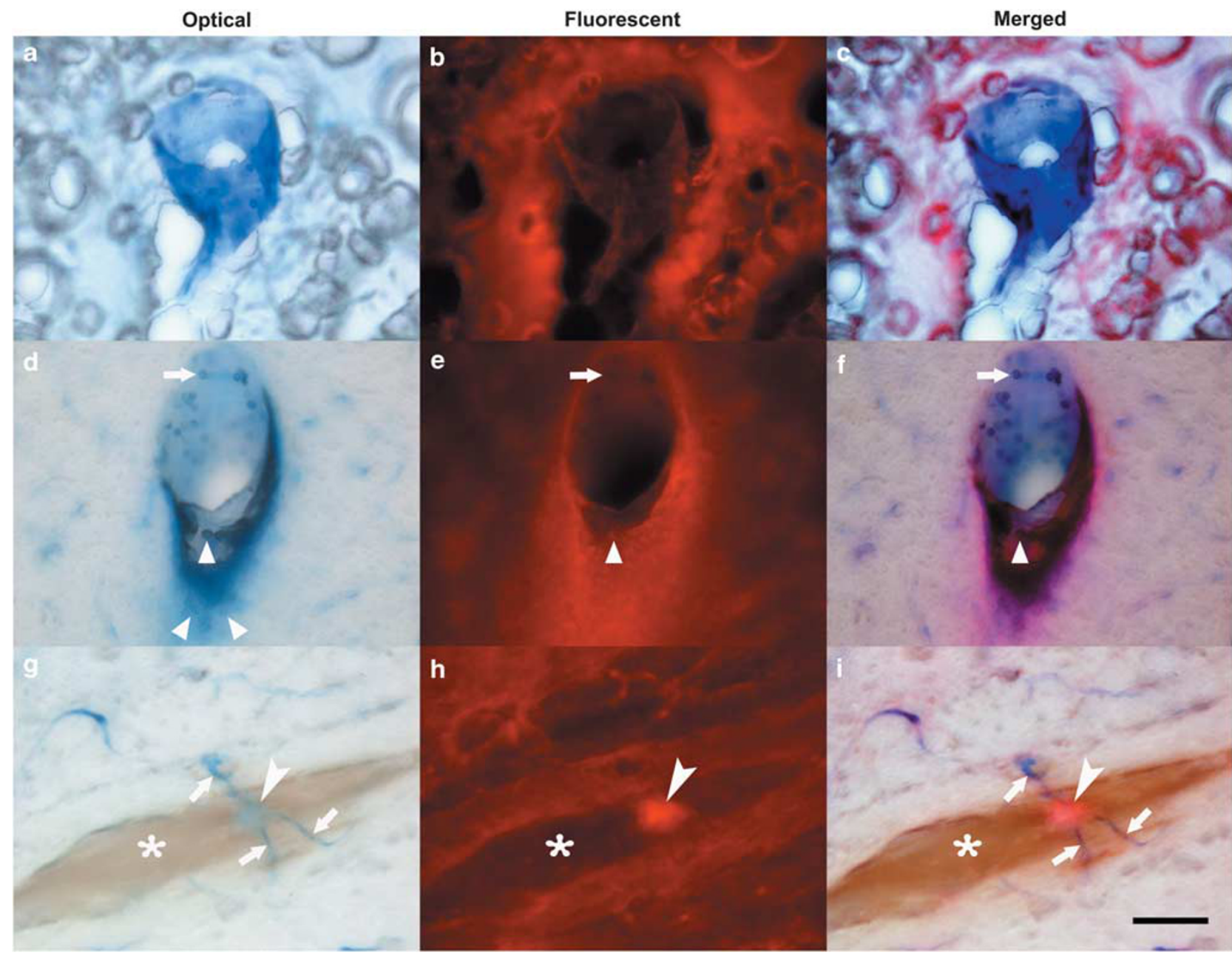

Figure 1 Representative images of the rat cerebral blood vessels perfused with the Evans Blue. In the normal rat, a cerebral blood vessel stained with the Evans Blue is in light blue as observed under the optical microscope (a) and emits weak red fluorescence under the fluorescence microscope, although slight fluorescence is observed around the vessel in the normal brain, which may be caused by the diffusion of the Evans Blue dye out of the blood vessel because of the air drying of the sections (b). In contrast, in the injured brain, a blood vessel in light blue is surrounded by a dark blue layer (d), which emits strong red fluorescence under the fluorescence microscope (e) and is a result of blood leakage because the exuded blood cells (d and $\mathbf{e}$, arrowheads) have the similar staining pattern to the blood cell inside the vessel (d and $\mathbf{e}$, arrow). This characteristic of the Evans Blue is confirmed by the images in ( $\mathbf{g})$ and (h), in which the leaked Evans Blue (g, arrowhead), from a broken blood vessel (g, arrows), emits strong fluorescence (h, arrowhead) when binding to the blood extravasation ( $\mathbf{g}$ and $\mathbf{h}$, asterisks). The images in (a) and (b), (d) and (e), and (g) and (h) are digitally combined in (c), (f), and (i), respectively, to show colocalization. (a-c) Uninjured brain sections. (d-i) Injured brain sections. Scale bar $=50 \mu \mathrm{m}$.

\section{Change in the Ratio of the Evans Blue-Perfused Capillary Number is Correlated with that of the MRI Signal Intensity after TBI}

As stated above, both the compromised ratio of the Evans Blue-perfused capillary number right after trauma and the MRI signal intensity were increased during the development of TBI (Figures 3 and 5). To evaluate the relationship between these two variables, the correlation analysis was performed. We found that there was a significant positive correlation between the changes in the ratio of the number of the Evans Blue-perfused capillary and the MRI signal intensity. The Pearson product-moment correlation coefficient $r=0.981$, $P=0.019$ (Figure 6).
MMP-9 Activity is Increased in the Early FP-Injured Brain To evaluate the activity of the MMP-9 in the early FP-injured brain, we detected the MMP-9 activities by gelatin zymography using the brain tissues from the normal rats and the injured rats at $0,1,4,12$, and $24 \mathrm{~h}$ after injury. In both the ipsilateral and the contralateral hemispheres, the MMP-9 activities started to increase at $4 \mathrm{~h}$, lasting to $24 \mathrm{~h}$ post trauma (Figure 7a and b). In the ipsilateral hemispheres, the MMP-9 activities were significantly increased at 12 and $24 \mathrm{~h}$ compared with that at either $0 \mathrm{~h}$ or $1 \mathrm{~h}$ post trauma (Figure $7 \mathrm{c}$, $P=0.002$ and 0.032 by Games-Howell post-hoc test, respectively, or Figure $7 \mathrm{c}, P=0.003$ by Games-Howell post-hoc test and $P=0.014$ by Student's $t$-test, respectively). Although no 

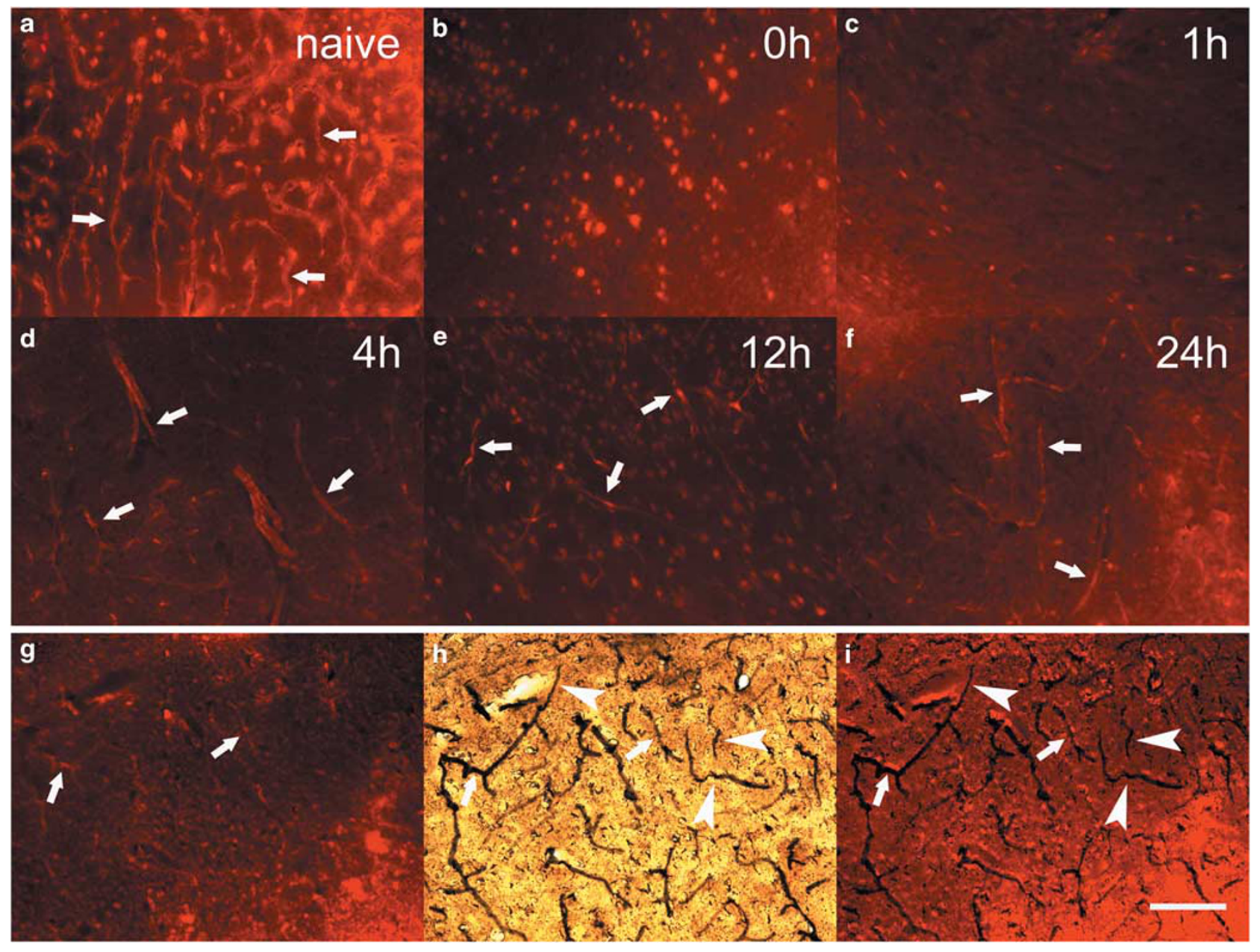

Figure 2 Autoregulation of the cerebral microvascular perfusion signaled by the Evans Blue and the alkaline phosphatase (AP) staining. The Evans Blue-perfused cerebral blood vessels (arrows) are clearly observed in the uninjured cortex under the fluorescence microscope (a). However, there are few vessels at the ischemic centers of the lesioned cortices at $0 \mathrm{~h}(\mathbf{b})$ and $1 \mathrm{~h}(\mathbf{c})$ after traumatic brain injury (TBI), which indicates the closure of the blood vessels and compromised capillary perfusion. In contrast, more blood vessels (arrows) are noted at $4 \mathrm{~h}$ (d), $12 \mathrm{~h}$ (e), and $24 \mathrm{~h}$ (f) post trauma. To show the perfused and unperfused vessels, in the same section of the ischemic center in the lesioned area of the brain at $4 \mathrm{~h}$ after TBI, the images with the Evans Blue labeling $(\mathbf{g})$ and with the AP staining (h) are merged in (i). It is noted that some AP-stained blood vessels (dark in color because of the $15 \mathrm{~min}$ reaction) are superimposed with the Evans Blue-perfused vessels (arrows), but others (arrowheads) are not (g-i), which clearly demonstrates that not all cerebral microvessels can be perfused by the Evans Blue dye at $4 \mathrm{~h}$ post trauma. (a) Uninjured brain section. (b-i) Injured brain sections. Scale bar $=100 \mu \mathrm{m}$.

significant augmentation of the MMP-9 activity in the lesioned hemisphere was detected at $4 \mathrm{~h}$ as compared with those at 0 and $1 \mathrm{~h}$ after injury, the increase was much greater at the $4 \mathrm{~h}$ time point (Figure $7 \mathrm{c}$ ). Likewise, there was no significant elevation in the activity of the MMP-9 in the contralateral hemisphere (Figure $7 \mathrm{~d}$ ).

\section{DISCUSSION}

In this study, we show the changes in the cerebral microvascular perfusion, BBB permeability, and MMP-9 activity over a 24-h period after TBI and find their dramatic alterations at $4 \mathrm{~h}$ post trauma. Interestingly, we note a significant positive correlation between the changes of the cerebral capillary perfusion and the BBB permeability, that is, the elevation of the $\mathrm{BBB}$ permeability is related to the increase of the blood vessel perfusion. Our findings indicate that the time point of $4 \mathrm{~h}$ post trauma may play a key role during the development of TBI and extend the MRI evaluation on BBB breakdown to $24 \mathrm{~h}$ in the lateral FP brain injury model of TBI in the rat. Importantly, the analysis of the positive correlation may guide us how to treat TBI.

\section{Cerebral Microvascular Perfusion Signaled by the Evans Blue Fluorescence}

The Evans Blue binds with high affinity to proteins, especially to albumin. When the Evans Blue binds with proteins, a vivid red fluorescent complex is formed. ${ }^{25,30}$ The Evans Blue has been used for the evaluation of the capillary perfusion by the intravenous administration of the tracer. ${ }^{19,21}$ In this study, we assessed the cerebral capillary perfusion by the ratio of the 
intracardiac Evans Blue-perfused microvessel number to the number of AP-stained total blood vessels in the rat brain.

There were several reasons for us to choose the combined methodology to evaluate the microvasculature perfusion in

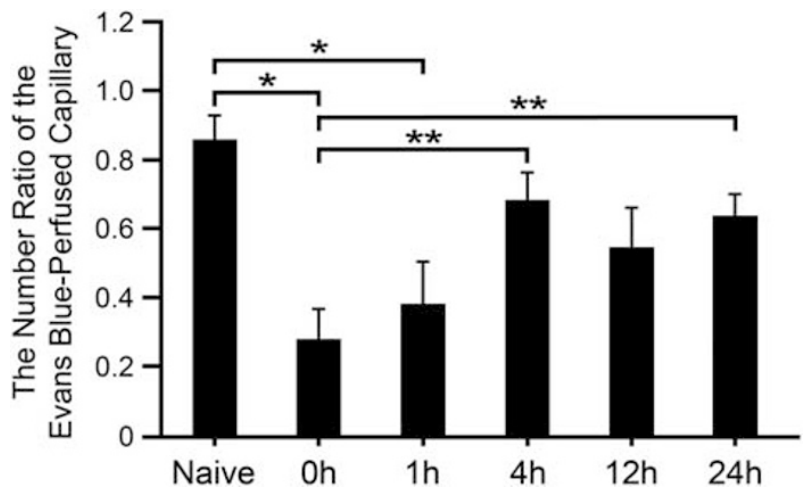

Figure 3 Statistic analyses of the ratios of the numbers of the Evans Blue-perfused capillaries to the alkaline phosphatase (AP)-stained microvessels in the cortices before and after fluid percussion (FP) injury. The ratios of the numbers of the Evans Blue-perfused capillary are significantly reduced in the lesioned cortices at 0 and $1 \mathrm{~h}$ post trauma compared with that in the normal cortex, which, however, dramatically recovered in the injured brains at 4 and $24 \mathrm{~h}$ after traumatic brain injury (TBI). ${ }^{*} P=0.005$ and 0.023 for 0 and $1 \mathrm{~h}$ post trauma versus normal, respectively, Tukey's post-hoc test. ${ }^{* *} P=0.016$ and 0.017 for 0 versus $4 \mathrm{~h}$ and $24 \mathrm{~h}$ after injury, respectively, Student's $t$-test. $n=4$ for each group. the brain. First, after the Evans Blue is intravenously administered, the different circulation times of the tracer may generate the different numbers of the Evans Blue-perfused microvessels in the injured brain, for example, with a longer circulation period of the tracer, larger amounts of the perfused capillaries are found both in the ischemic center and in the pericontusional area as compared to the ones with a shorter time of the circulation, although the period does not affect the vessel counts in the normal brain. ${ }^{21}$ In contrast, no circulation time is needed in our study. Second, unfixed brains are generally used for the sections when the tracer is given intravenously. ${ }^{19,21}$ In our preliminary experiments, we found that the unfixed brain tissue was much looser than the PFA-perfused brain and that the sections from the former were larger than those from the latter. As a result, the size of the microvascular lumen in the unfixed section might be increased, which then affects the capillary counts. Third, our intracardiac approach can imitate the physical conditions, such as producing the normal CPP, as described above. Fourth, the intracardiac Evans Blue-perfused cerebral blood vessels clearly illustrated the morphology of the vessels as shown in Figure 2. Fifth, the PFA-fixed brain section can still be used for the AP staining of the blood vessels, and the ratio between the perfused capillary number and total microvessel amount reflects the cerebral capillary perfusion better than the absolute number of the perfused capillary alone does.

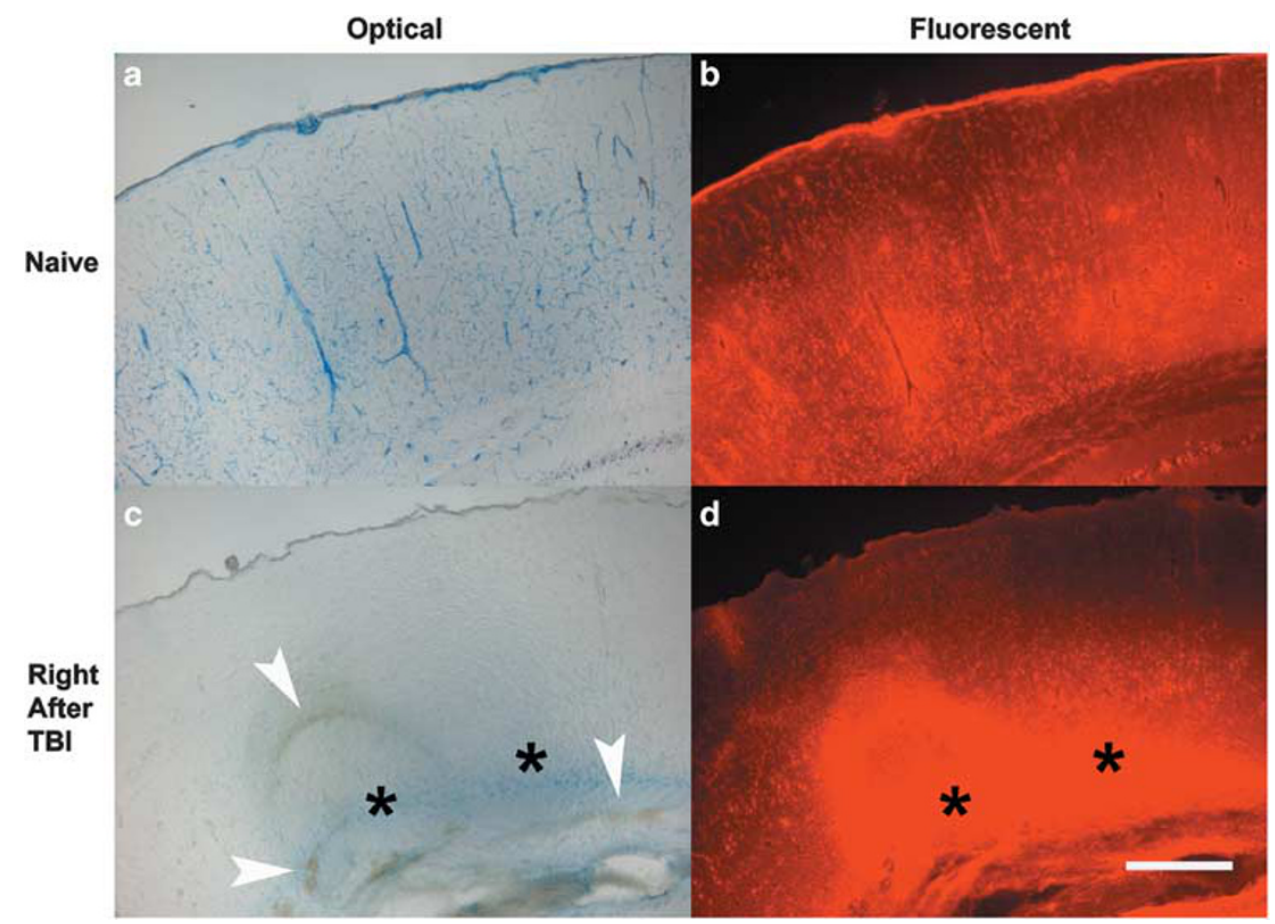

Figure 4 Primary mechanical blood-brain barrier (BBB) disruption immediately after traumatic brain injury (TBI) measured by intracardially perfused Evans Blue. In the normal animal, the Evans Blue clearly labels the cerebral blood vessels (a and b). In contrast, after TBI, hemorrhages (arrowheads) are visible in the injured area of the brain (c). At the non-hemorrhagic sites (asterisks) of the injured cortex, strong red fluorescence is noted (c and $\mathbf{d}$ ), which indicates the extravasation of the Evans Blue dye across the BBB. (a, b) Uninjured brain sections. (c, d) Injured brain sections. Scale bar $=400 \mu \mathrm{m}$. 


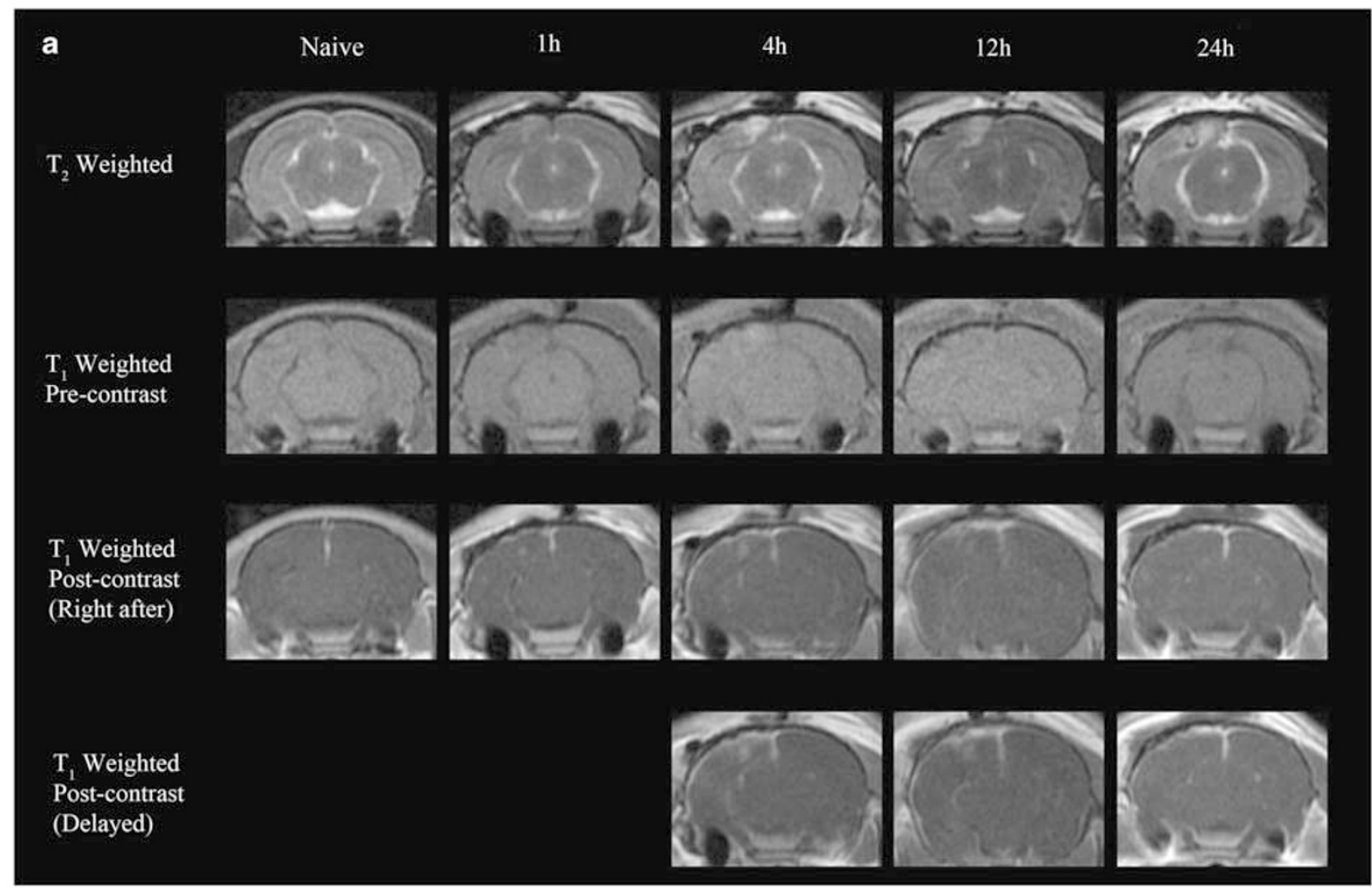

b

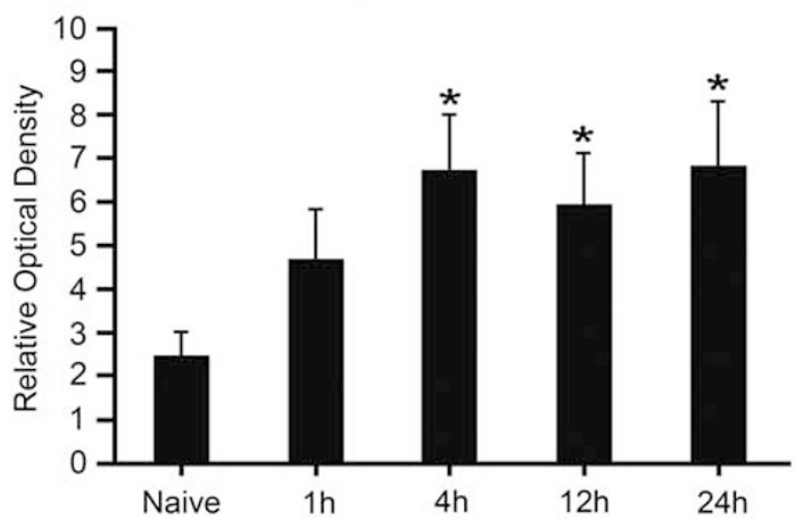

c

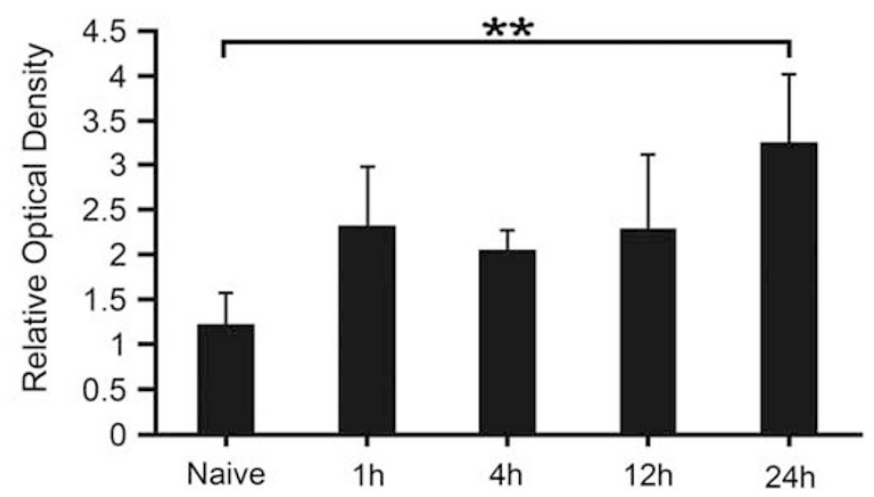

Figure 5 Magnetic resonance imaging (MRI)-monitored blood-brain barrier (BBB) breakdown after lateral fluid percussion (FP) brain injury. The BBB disruption is detected by the MRI with the contrast agent Gd-DTPA. The series of the representative MRI images are from the same animal to compare one another easily, whereas the statistic analyses of the optical densities (Gd-DTPA extravasation) are based on the MRI data from six animals. On the $\mathrm{T}_{2}$-weighted MRI images, edematous and lesioned areas are clearly exhibited in the ipsilateral cortices of the injured brains at $1,4,12$, and $24 \mathrm{~h}$ post trauma (a). There are fundamental signal intensities in the uninjured and injured brains on the pre-contrast $\mathrm{T}_{1}$-weighted MRI images, although slight enhancement is viewed in the injured area of the brain at $4 \mathrm{~h}$ post impact (a). After the injection of the contrast agent Gd-DTPA, the signal intensities are significantly greater in the ipsilateral cortices and hippocampi (the left sides of the MRI images) at 4,12 , and $24 \mathrm{~h}$ (a and $\mathbf{b}$ ), as well as in the contralateral brain (the right side) at $24 \mathrm{~h}$ (a and $\mathbf{c}$ ) after impact compared with that in the normal brain on the $\mathrm{T}_{1}$-weighted MRI images. ${ }^{*} P=0.021$, 0.013 , and 0.027 for 4,12 , and $24 \mathrm{~h}$ post trauma versus normal, respectively, one-way analysis of variance (ANOVA) with repeated measures. ${ }^{* *} P=0.05$, Student's $t$-test. $n=6$ for each group.

Thus, the above reasons convince us that the intracardiac administration of the Evans Blue, combined with AP staining, is able to evaluate the change of the cerebral microvascular perfusion after injury. In fact, the number of the perfused capillaries calculated with the intracardiac Evans
Blue in our study was similar to the one counted with the intravenous Evans Blue reported by Göbel et al ${ }^{19}$ (369 \pm 40 vs $339 \pm 25$ counts per $\mathrm{mm}^{2}$ in the uninjured rats).

Under both the optical and the fluorescence microscopes, we found that unlike in the uninjured brain, the perfused 
Evans Blue was only able to label few cerebral microvessels in the impacted cortices at 0 and $1 \mathrm{~h}$ post trauma, which may indicate the closure of the microvessels and the compromised

\section{Correlation}

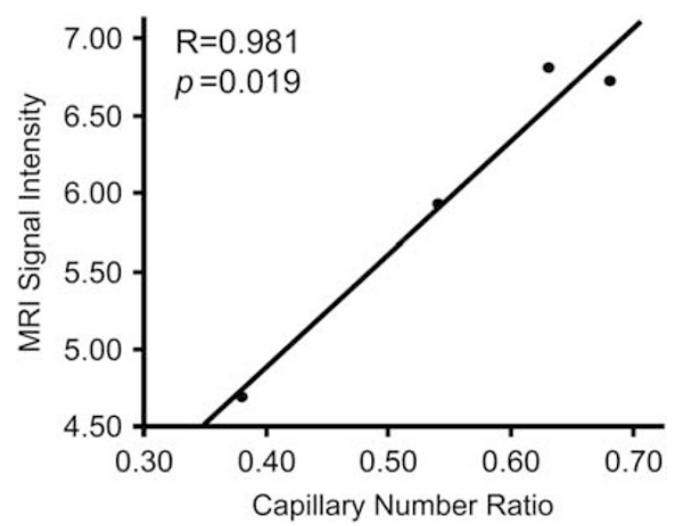

Figure 6 Correlation analysis between the changes in the ratio of the Evans Blue-perfused capillary number and the MRI signal intensity. As the ratio of the perfused capillary number increases, so does the MRI signal intensity (Pearson's correlation test, correlation coefficient $r=0.981, P=0.019$ ). capillary perfusion at the lesioned sites in response to impact. A series of factors, such as vasospasm, ${ }^{31}$ intracranial hypertension, ${ }^{32}$ and decreased $\mathrm{CPP},{ }^{33}$ may contribute to the compromised perfusion after TBI. In our study, we observed that the blood clots within the cerebral microvessels hindered the capillary perfusion (data not shown).

At $4 \mathrm{~h}$ after FP injury, however, more fluoresced blood vessels were visualized in the impacted area. The ratios of the numbers of the Evans Blue-perfused capillaries were significantly greater at 4 and $24 \mathrm{~h}$ as compared to that at $0 \mathrm{~h}$ post trauma. Our results imply that the moment of $4 \mathrm{~h}$ post trauma may be a critical turning point in terms of the capillary perfusion in response to FP injury. Two reasons might account for the increase in the ratio of the Evan Blue-perfused vessel number from 1 to $4 \mathrm{~h}$ post trauma: (1) the closed cerebral capillaries post-trauma re-open. (2) The intravascular clotting, as mentioned above, and the gradual sealing of the injured vessels after TBI might increase upstream blood pressure and further build a bypass to the ischemic area. Lundblad et $a l^{34}$ have reported a progressive reduction in the perfused capillary numbers at 3 and $24 \mathrm{~h}$ post trauma in a mouse controlled cortical impact (CCI) model of TBI, which is

Ipsilatera

Naive

Oh

1h

$4 h$

$12 \mathrm{~h}$

$24 \mathrm{~h}$

Pro MMP-9

Pro MMP-2
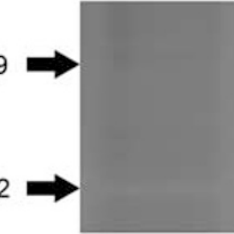

b

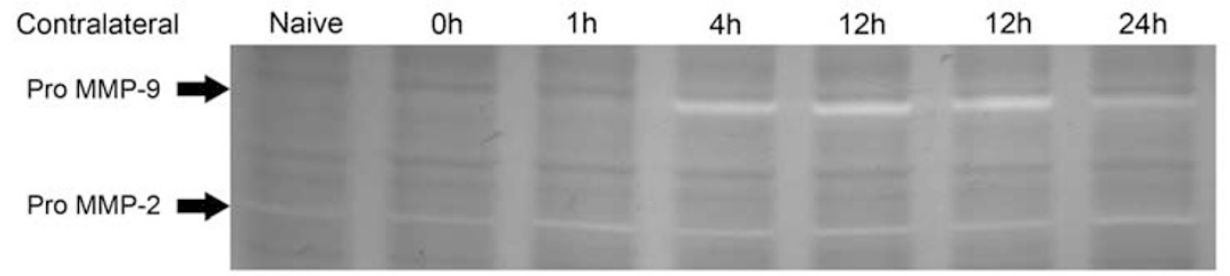

C

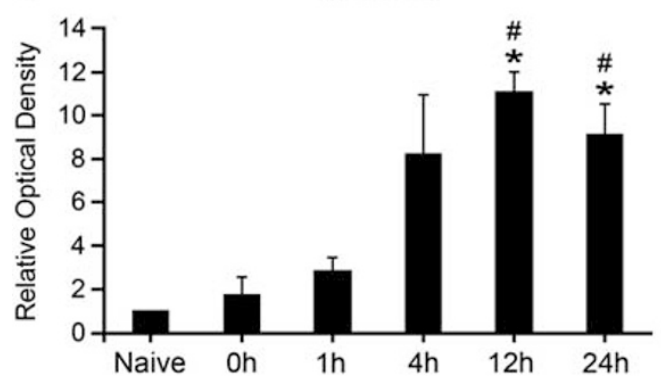

d

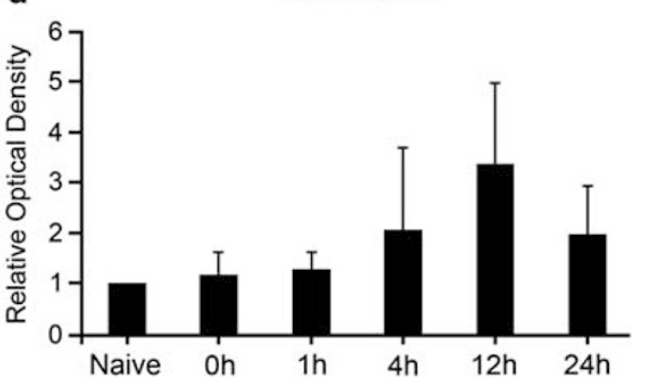

Figure 7 Time course of the matrix metalloproteinase-9 (MMP-9) expression in the early fluid percussion (FP)-injured rat brain measured by gelatin zymography. In both the ipsilateral and the contralateral hemispheres, the increases of the MMP-9 activity start at $4 \mathrm{~h}$ and maintained to 12 and $24 \mathrm{~h}$ post trauma ( $\mathbf{a}$ and $\mathbf{b}$ ). Compared with the MMP-9 activities at 0 and $1 \mathrm{~h}$ post trauma, the ones in the ipsilateral hemispheres are significantly greater at 12 and $24 \mathrm{~h}$ after injury (c). However, no significant elevation of the MMP-9 activity is detected in the contralateral hemisphere of the injured brain (d). ${ }^{*} P=0.002$ and 0.032 for 12 and $24 \mathrm{~h}$ versus $0 \mathrm{~h}$ after injury, respectively, Games-Howell post-hoc test. ${ }^{\#} P=0.003$ for 12 versus $1 \mathrm{~h}$ post trauma, Games-Howell post-hoc test; 0.014 for 24 versus $1 \mathrm{~h}$ after injury, Student's $t$-test. $n=4$ for each group in the ipsilateral hemisphere; 3 per group of naïve, 0,1 , or $4 \mathrm{~h}$, as well as 4 per group of 12 or $24 \mathrm{~h}$ post lesion in the contralateral hemisphere. 
different from our findings in the rat FP brain injury model. The discrepancy might be caused by the difference of the trauma intensities, TBI models, or animal species. Park et al $b^{35}$ have documented the diminishment of the microvascular densities in the rat brain at $24 \mathrm{~h}$ and 2 weeks after two intensities of FP brain trauma. ${ }^{35}$ In contrast, we evaluated the change of the microvascular perfusion in the early FP-injured brains of the rat. In the clinic, since earlier therapy yields better outcomes, the patients could profit from our finding.

\section{Primary BBB Breakdown Right after Injury}

As the CNS trauma causes both the primary and the secondary injury, the $\mathrm{BBB} /$ spinal cord barrier correspondingly undergo two phases of the breakdown: primary breakdown and secondary breakdown. ${ }^{36,37}$ Some studies have addressed that the primary barrier breakdown is detected by the in vivo perfusion of tracers before injury, ${ }^{11,37}$ but this evaluation on the barrier permeability might not indeed reflect the barrier disruption right after TBI. In our study, we intracardially perfused the Evans Blue immediately after injury to measure the primary $\mathrm{BBB}$ disruption, which may guide the intravenous administration of medicines right after post trauma. We found that the BBB permeability to the tracer was augmented immediately after post trauma, which is consistent with a previous study showing that the Evans Blue is extravasated in the mouse brain right after cryoinjury. ${ }^{25} \mathrm{As}$ the blood was depleted by saline before the intracardiac perfusion of the Evans Blue, our method avoids the interaction between the Evans Blue and the plasma proteins such as albumin. Therefore, our finding indicates the BBB leakage only to the Evans Blue.

\section{Impaired BBB in the Early Injured Brains Evaluated by MRI}

Many protocols have been reported to evaluate the breakdown of the BBB. ${ }^{9,10,12}$ For example, the extraction of the extravasated Evans Blue from the brain tissue has been used to assess quantitatively the barrier impairment. ${ }^{38,39}$ In our study, we used the MRI measurement to detect the BBB disruption after TBI because this technique is widely applied in the clinic to diagnose the CNS diseases and patients could potentially benefit from our research work.

The MRI contrast agent Gd-DTPA is commonly used to make brain structures good contrast. In the uninjured brain, the Gd-DTPA cannot go through the BBB. However, when the barrier is disrupted, the contrast agent is extravasated, which can enhance the signal intensity on the MRI image. ${ }^{13,40}$ There is evidence that the BBB breakdown evaluated by the MRI varies in different experimental TBI models. ${ }^{13,41}$ Beaumont et $a l^{41}$ have reported that in the rat impact acceleration model (IAM) of TBI, the BBB rapidly opens and then fast closes to $36 \mathrm{~h}$ after injury, whereas the BBB permeability is increased for $4 \mathrm{~h}$ in the CCI TBI model of the rat.

In this study, we monitored the BBB disruption using the MRI in the lateral FP brain injury model of TBI in the rat. We found that the MRI signal intensities were significantly increased in the ipsilateral cortices and hippocampi at 4, 12, and $24 \mathrm{~h}$ after injury, as well as in the contralateral part at $24 \mathrm{~h}$ post trauma as compared with those in the normal control areas. Two reasons might be related to the elevation of the MRI signal intensity: one is an increase in the amount of the contrast agent within the blood vessels because of the increased number of the perfused capillaries and/or the augmented blood flow, and the other is more tissue uptake of the tracer under the condition of the BBB breakdown. In our study, because the ratio of the Evans Blue-perfused capillary number was correspondingly decreased in the impacted area at each time point after TBI compared with that in the normal brain as described above, we conclude that the augmentations of the MRI signal intensities at 4,12 , and $24 \mathrm{~h}$ after TBI are due to the BBB leakage to the contrast agent. There is a previous report that by using the MRI technique, the disruption of the BBB is found to retain up to $12 \mathrm{~h}$ in the rat lateral FP brain injury model. ${ }^{14}$ Therefore, our study extends the MRI evaluation on the BBB breakdown to $24 \mathrm{~h}$ after FP brain injury in the rat. Our findings are consistent with the histological investigations not only from our study that the BBB is disrupted right post trauma but also from other previous reports that the barrier impairment lasts days after TBI. ${ }^{42,43}$

We detected that the changes of both the MRI signal intensity and the perfused capillary number ratio in the ipsilateral part of the injured brain were more prominent at $4 \mathrm{~h}$ than those at $1 \mathrm{~h}$ after impact. To fathom which of the factors mentioned above contributes to the elevation of the MRI signal intensity, the tracer leakage on the MRI image was observed. On account of the fact that our MRI image exhibited more tracer leakage in the impaired area at $4 \mathrm{~h}$ than that at $1 \mathrm{~h}$ post trauma, we conclude that the greater MRI signal intensity at $4 \mathrm{~h}$ is at least partly due to more tracer crossing the disrupted $\mathrm{BBB}$ as compared with that at $1 \mathrm{~h}$ after injury. Our finding of the significantly increased BBB permeability at $4 \mathrm{~h}$ post trauma is in accordance with a previous report that the barrier leakage to $\alpha$-aminoisobutyric acid peaks at $4 \mathrm{~h}$ after a focal cortical contusion. ${ }^{44}$ The elevation of the $\mathrm{BBB}$ permeability between 1 and $4 \mathrm{~h}$ following injury might be due to further damage of the disrupted barrier post trauma, which is caused by the ischemia right after $\mathrm{TBI},{ }^{44}$ or the greater surface area of the leaky BBB induced by the higher perfusion of the disrupted vessels via the above mentioned bypass.

Our $\mathrm{T}_{2}$-weighted MRI images showed that there were relative larger edematous and lesioned areas in the injured brains at 4,12 , and $24 \mathrm{~h}$ post trauma than the ones in the normal brain and in the injured brain at $1 \mathrm{~h}$ after TBI, which were in agreement with the significant increases in the BBB permeability at these time points post trauma. Traumatic brain edema has been regarded as a combined event of vasogenic and cellular edemas, although the cellular factor is predominant. ${ }^{41,45}$ Thus, our findings may imply a vasogenic factor in the edema formation after TBI. 


\section{Relationships between the Changes of the Capillary} Perfusion, BBB Breakdown, and MMP-9 Activity after TBI

We noted that there was a significant positive correlation between the ratio of the Evans Blue-perfused capillary number, reflecting capillary perfusion, and the MRI signal intensity, representing the BBB permeability, from 1 to $24 \mathrm{~h}$ post trauma. Although this correlation cannot determine a cause-and-effect relationship between two variables, it indicates that the change in the BBB permeability is related to the alteration of the capillary perfusion and that as the cerebral capillary perfusion increased so did the BBB permeability, that is, the increase in the BBB permeability is related to the restoration of the perfusion over a 24 -h period after injury. Our finding may guide the TBI therapy. For example, since the cerebral capillary perfusion was compromised right after injury, if any therapeutic strategy, such as the fluid administration, to improve the microvascular perfusion is conducted at the beginning of TBI, the treatment should be paid more attention at 4 and $24 \mathrm{~h}$ post trauma because it may cause or aggravate the brain edema under the condition of the augmented BBB permeability at these time points after TBI. In fact, our MRI finding indicated that the elevation of the barrier permeability was consistent with the edema in the injured brain as mentioned before.

We found that the MMP-9 enzymatic activity was augmented after FP injury. This finding is in agreement with other previous studies in which the activity of the MMP- $9^{10}$ and its mRNA expression ${ }^{46}$ have been increased in the rat CCI model of TBI. It has been reported that the BBB disruption may be associated with the MMP-9 activity. ${ }^{10,15}$ Some studies have documented that the MMP activity inhibitors are able to attenuate the BBB breakdown. ${ }^{15,47}$ In our study, we noted that there was a similar pattern in the augmentations of the MMP-9 activity and the BBB permeability after TBI. Therefore, our finding may contribute to the investigation of their relationship.

\section{CONCLUSIONS}

The purpose of our study is to explore the correlation between the changes of the cerebral microvascular perfusion and the BBB permeability after TBI. Thus, we evaluated the alterations of the cerebral capillary perfusion by the intracardiac perfusion of the Evans Blue and the BBB permeability by the MRI during a period of $24 \mathrm{~h}$ post trauma in the lateral FP brain injury model of TBI in the rat. Moreover, because the MMP-9 activity may be related to the BBB disruption, the gelatin zymography was used to assess the MMP-9 activity after injury. We found the great changes at $4 \mathrm{~h}$ post trauma in the ratio of the Evans Blue-perfused cerebral microvessel number, the BBB permeability, and the MMP-9 activity, as well as a significant positive correlation between the changes of the cerebral microvascular perfusion and the BBB permeability post trauma. Therefore, our findings demonstrate that the time point of $4 \mathrm{~h}$ post trauma may play a key role during the development of TBI and that the MRI can be used to observe the long event of the BBB breakdown in the rat lateral FP brain injury model of TBI. Importantly, the correlation analysis may guide us how to optimize the cerebral capillary perfusion, but not to cause other neurological pathophysiologies such as the edema.

\section{ACKNOWLEDGEMENTS}

This work was supported by the Grants of National Science and Nature Committee of China (No. 30973086), National Health Ministry of China (No. 200802093), Shanghai Science and Technology Committee (No. 10JC1409800), and Program for Shanghai Outstanding Medical Academic Leader.

\section{DISCLOSURE/CONFLICT OF INTEREST}

The authors declare no conflict of interest.

1. Lindsay KW, McLatchie G, Jennett B. Serious head injury in sport. BMJ 1980;281:789-791.

2. Ghajar J. Traumatic brain injury. Lancet 2000;356:923-929.

3. Dewey CW. Emergency management of the head trauma patient. Vet Clin N Am Small Anim Pract 2000;30:207-225.

4. Shackford SR, Mackersie RC, Davis JW, et al. Epidemiology and pathology of traumatic deaths occurring at a Level I Trauma Center in a regionalized system: the importance of secondary brain injury J Trauma 1989:29:1392-1397.

5. Werner $\mathrm{C}$, Engelhard K. Pathophysiology of traumatic brain injury. Br J Anaesth 2007;99:4-9.

6. Sande A, West C. Traumatic brain injury: a review of pathophysiology and management. J Vet Emerg Crit Care (San Antonio) 2010;20: 177-190.

7. Janzer RC, Raff M. Astrocytes induce blood-brain barrier properties in endothelial cells. Nature 1987;325:253-257.

8. Kniesel $\mathrm{U}$, Wolburg H. Tight junctions of the blood-brain barrier. Cell Mol Neurobiol 2000;20:57-76.

9. Duvdevani R, Roof RL, Fulop Z, et al. Blood-brain barrier breakdown and edema formation following frontal cortical contusion: does hormonal status play a role?. J Neurotrauma 1995;12:65-75.

10. Shigemori $Y$, Katayama Y, Mori T, et al. Matrix metalloproteinase-9 is associated with blood-brain barrier opening and brain edema formation after cortical contusion in rats. Acta Neurochir Suppl 2006;96:130-133.

11. Fukuda $\mathrm{K}$, Tanno $\mathrm{H}$, Okimura $\mathrm{Y}$, et al. The blood-brain barrier disruption to circulating proteins in the early period after fluid percussion brain injury in rats. J Neurotrauma 1995;12:315-324.

12. Habgood MD, Bye N, Dziegielewska KM, et al. Changes in blood-brain barrier permeability to large and small molecules following traumatic brain injury in mice. Eur J Neurosci 2007;25:231-238.

13. Ford JC, Wood AK, Van Winkle TJ, et al. Magnetic resonance imaging observations of blood-brain-barrier permeability in an animal model of brain injury. Acad Radiol 1997;4:115-126.

14. Schneider G, Fries P, Wagner-Jochem D, et al. Pathophysiological changes after traumatic brain injury: comparison of two experimental animal models by means of MRI. MAGMA 2002;14:233-241.

15. Yamaguchi $M$, Jadhav $V$, Obenaus $A$, et al. Matrix metalloproteinase inhibition attenuates brain edema in an in vivo model of surgically-induced brain injury. Neurosurgery 2007;61:1067-1075, discussion 1075-1076.

16. Nilsson P, Gazelius B, Carlson H, et al. Continuous measurement of changes in regional cerebral blood flow following cortical compression contusion trauma in the rat. J Neurotrauma 1996;13: 201-207.

17. Hayward NM, Tuunanen PI, Immonen R, et al. Magnetic resonance imaging of regional hemodynamic and cerebrovascular recovery after lateral fluid-percussion brain injury in rats. J Cereb Blood Flow Metab 2011;31:166-177.

18. McGoron AJ, Capille M, Georgiou MF, et al. Post traumatic brain perfusion SPECT analysis using reconstructed ROI maps of radioactive 
microsphere derived cerebral blood flow and statistical parametric mapping. BMC Med Imag 2008;8:4.

19. Göbel U, Theilen H, Kuschinsky W. Congruence of total and perfused capillary network in rat brains. Circ Res 1990;66:271-281.

20. Zoccoli G, Lucchi ML, Andreoli E, et al. Brain capillary perfusion during sleep. J Cereb Blood Flow Metab 1996;16:1312-1318.

21. Dawson DA, Ruetzler CA, Hallenbeck JM. Temporal impairment of microcirculatory perfusion following focal cerebral ischemia in the spontaneously hypertensive rat. Brain Res 1997;749:200-208.

22. Clifton GL, Choi SC, Miller ER, et al. Intercenter variance in clinical trials of head trauma-experience of the National Acute Brain Injury Study: Hypothermia. J Neurosurg 2001;95:751-755.

23. Grände PO. The 'Lund Concept' for the treatment of severe head trauma-physiological principles and clinical application. Intens Care Med 2006;32:1475-1484.

24. Mclntosh TK, Vink $R$, Noble $L$, et al. Traumatic brain injury in the rat: characterization of a lateral fluid-percussion model. Neuroscience 1989:28:233-244.

25. del Valle J, Camins A, Pallas $M$, et al. A new method for determining blood-brain barrier integrity based on intracardiac perfusion of an Evans Blue-Hoechst cocktail. J Neurosci Methods 2008;174:42-49.

26. Weiss HR. Measurement of cerebral capillary perfusion with a fluorescent label. Microvasc Res 1988;36:172-180.

27. Barth $\mathrm{KN}$, Onesti ST, Krauss WE, et al. A simple and reliable technique to monitor intracranial pressure in the rat: technical note. Neurosurgery 1992;30:138-140.

28. Wang-Fischer Y, Koetzner L. Endpoints for stroke studies. In: WangFischer Y (ed) Manual of Stroke Models in Rats, Boca Raton, FL, 2009 pp193-221.

29. Kroppenstedt SN, Kern M, Thomale UW, et al. Effect of cerebral perfusion pressure on contusion volume following impact injury. J Neurosurg 1999;90:520-526.

30. Hawkins BT, Egleton RD. Fluorescence imaging of blood-brain barrier disruption. J Neurosci Methods 2006;151:262-267.

31. Oertel M, Boscardin WJ, Obrist WD, et al. Posttraumatic vasospasm: the epidemiology, severity, and time course of an underestimated phenomenon: a prospective study performed in 299 patients J Neurosurg 2005;103:812-824.

32. Baethmann A, Eriskat J, Stoffel M, et al. Special aspects of severe head injury: recent developments. Curr Opin Anaesthesiol 1998; 11:193-200.

33. Smith MJ, Hartl R. Maintaining cerebral perfusion after polytrauma using arginine vasopressin: a classic drug revisited. Crit Care Med 2008;36:2703-2704.

34. Lundblad C, Grände PO, Bentzer P. A mouse model for evaluation of capillary perfusion, microvascular permeability, cortical blood flow, and cortical edema in the traumatized brain. J Neurotrauma 2004 21:741-753.

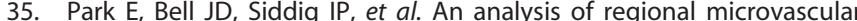
loss and recovery following two grades of fluid percussion trauma: a role for hypoxia-inducible factors in traumatic brain injury. J Cereb Blood Flow Metab 2009;29:575-584.

36. Kita $\mathrm{T}$, Liu L, Tanaka $\mathrm{N}$, et al. The expression of tumor necrosis factoralpha in the rat brain after fluid percussive injury. Int J Legal Med 1997;110:305-311.

37. Maikos JT, Shreiber DI. Immediate damage to the blood-spinal cord barrier due to mechanical trauma. J Neurotrauma 2007;24:492-507.

38. Uyama O, Okamura N, Yanase $\mathrm{M}$, et al. Quantitative evaluation of vascular permeability in the gerbil brain after transient ischemia using Evans Blue fluorescence. J Cereb Blood Flow Metab 1988;8: 282-284

39. Rancan M, Morganti-Kossmann MC, Barnum SR, et al. Central nervous system-targeted complement inhibition mediates neuroprotection after closed head injury in transgenic mice. J Cereb Blood Flow Metab 2003;23:1070-1074.

40. Beaumont A, Marmarou A, Fatouros $P$, et al. Secondary insults worsen blood brain barrier dysfunction assessed by MRI in cerebral contusion. Acta Neurochir Suppl 2002;81:217-219.

41. Beaumont A, Marmarou A, Hayasaki K, et al. The permissive nature of blood brain barrier (BBB) opening in edema formation following traumatic brain injury. Acta Neurochir Suppl 2000;76:125-129.

42. Tanno H, Nockels RP, Pitts LH, et al. Breakdown of the blood-brain barrier after fluid percussive brain injury in the rat. Part 1: Distribution and time course of protein extravasation. J Neurotrauma 1992;9:21-32.

43. Lotocki G, Vaccari JP, Perez ER, et al. Alterations in blood-brain barrier permeability to large and small molecules and leukocyte accumulation after traumatic brain injury: effects of post-traumatic hypothermia. J Neurotrauma 2009;26:1123-1134.

44. Mathew $P$, Bullock $R$, Teasdale $G$, et al. Changes in local microvascular permeability and in the effect of intervention with 21-aminosteroid (Tirilazad) in a new experimental model of focal cortical injury in the rat. J Neurotrauma 1996;13:465-472.

45. Marmarou A, Signoretti S, Aygok G, et al. Traumatic brain edema in diffuse and focal injury: cellular or vasogenic?. Acta Neurochir Suppl 2006;96:24-29.

46. Hayashi T, Kaneko Y, Yu S, et al. Quantitative analyses of matrix metalloproteinase activity after traumatic brain injury in adult rats. Brain Res 2009;1280:172-177.

47. Higashida T, Kreipke CW, Rafols JA, et al. The role of hypoxia-inducible factor- $1 \alpha$, aquaporin- 4 , and matrix metalloproteinase-9 in blood-brain barrier disruption and brain edema after traumatic brain injury. J Neurosurg 2011;114:92-101. 